\title{
Messung und Simulation der Erwärmung von ermüdungsbeanspruchten Betonprobekörpern
}

\author{
Albert Vogela, Conrad Völkera, Matthias Bodeb, Steffen Marx ${ }^{b}$ \\ ${ }^{a}$ Bauhaus-Universität Weimar, Professur Bauphysik, Coudraystraße 11a, 99423 Weimar, Deutschland \\ ${ }^{b}$ Leibniz Universität Hannover, Institut für Massivbau, Appelstraße 9a, 30167 Hannover, Deutschland
}

\begin{abstract}
Copyright-Vermerk
Copyright 2020 Ernst \& Sohn. Dieser Artikel kann für den persönlichen Gebrauch heruntergeladen werden. Andere Verwendungen bedürfen der vorherigen Zustimmung der Autoren und des Verlags Ernst \& Sohn.
\end{abstract}

Der folgende Artikel erschien in der Bauphysik 42 (2), 86-93 (2020).

\begin{abstract}
Im vorliegenden Beitrag werden Messungen und Berechnungen vorgestellt, die die Temperaturentwicklung in Betonzylindern aufgrund zyklischer Beanspruchung genau beschreiben. Die Messungen wurden in einem Versuchsstand, die Berechnungen im FEM-Programm ANSYS durchgeführt. Mit Hilfe der Temperaturmessungen konnten die Simulationen für die Temperaturentwicklung der Betonzylinder mit der verwendeten Betonrezeptur validiert werden. Die Untersuchungen lassen den Schluss zu, dass bei zyklischer Probekörperbelastung und der einhergehenden Probekörperdehnung Energie dissipiert wird und diese maßgeblich für die Erwärmung der Probe verantwortlich ist.

This paper presents measurements and simulations that describe the temperature development in concrete cylinders due to cyclic loading. The measurements were carried out in a test stand, the simulations in the FEM program ANSYS. The simulations of the temperature development in the concrete cylinders with the used concrete recipe were validated using the temperature measurements. The investigations lead to the conclusion that energy is dissipated during cyclic test specimen loading and the accompanying test specimen elongation and that this is mainly responsible for the heating of the specimen.
\end{abstract}

\section{Einleitung}

Bauwerke sind ständigen statischen und dynamischen Beanspruchungen ausgesetzt. Dynamische Beanspruchungen ergeben sich aus zeitlich variierenden Belastungsgrößen wie zum Beispiel Windoder Wellenangriff bei Offshore-Windkraftanlagen oder aber Schwerlastverkehr auf Brücken, woraus sich über die gesamte Lebenszeit des Bauwerks von bis $\mathrm{zu} 10^{9}$ Lastwechsel ergeben können [1]. Für diese dynamischen Beanspruchungen ist der Ermüdungswiderstand des Betons von Bedeutung, der durch Wöhlerversuche ermittelt wird, bei denen die Probekörper durch lastgesteuerte Ermüdungsversuche zyklisch mechanischer Beanspruchung ausgesetzt sind. Dabei wird Probekörpern aufgrund ihres viskoelastoplastischen Materialverhaltens Energie zugeführt. Diese teilt sich auf in einen reversiblen-elastischen, einen nichtreversiblen-plastischen sowie einen viskosen Anteil, der in der Probe zu einem großen Teil in Wärme umgewandelt wird. Die zugeführte Wärmeenergie ist von der Belastungsgröße, der Belastungsfrequenz, den Betonmaterialparametern sowie dem konvektiven und radiativen Wärmeübergang an die Umgebung (Raumluft, Umfassungsflächen) abhängig. Versuche zeigen, dass Belastungsfrequenzen von $\mathrm{f}_{\mathrm{p}}=10 \mathrm{~Hz}$ bereits $\mathrm{zu}$ hohen Probekörpertemperaturen von ca. $70^{\circ} \mathrm{C}$ [1] führen können. Durch diese Erwärmung des Betons kann es zum vorzeitigen Versagen der Probe kommen [1, 2]. So kann die Betonfestigkeit bei $100{ }^{\circ} \mathrm{C}$ gegenüber einer Temperatur von $20^{\circ} \mathrm{C}$ um $25 \%$ verringert sein, bei $80{ }^{\circ} \mathrm{C}$ um $15 \%$ [3]. Das bedeutet, dass die gegenwärtigen dynamischen Belastungsprüfungen mit sehr hohen Lastwechselzahlen und demzufolge hohen Betonprobekörpertemperaturen ungenau sind. Um zukünftig realitätsnahe Widerstandsgrößen für zyklische Beanspruchung zu erhalten, müssen die Ursachen und Einflussgrößen dieser übermäßigen Erwärmung mit geeigneten Methoden untersucht werden [4-7]. In aktuellen Untersuchungen wird die Hypothese vertreten, dass die Probekörpererwärmung durch dissipierte Energie erfolgt und die Erwärmung im Zusammenhang mit der Schädigung und dem Versagen steht [6, 7]. Um diese Hypothese zu untersuchen, wurden Messungen in einem Versuchsstand sowie numerische Simulationen durchgeführt, die folgend vorgestellt werden.

\section{Voruntersuchungen Materialparameter}

Für die numerischen Simulationen ist die möglichst genaue Kenntnis der Materialparameter 
unabdingbar. Aufgrund der starken Erwärmung des Betons konnten hier aber nicht die üblichen statischen Tabellenwerte verwendet werden. Daher wurden die für die Simulationen relevanten bauphysikalischen Parameter Wärmeleitfähigkeit $\lambda$, spezifische Wärmekapazität c und der Temperaturausdehnungskoeffizient $\alpha_{\mathrm{T}, \mathrm{B}}$ des Betons temperaturabhängig gemessen.

\subsection{Wärmeleitfähigkeit}

Die Wärmeleitfähigkeit $\lambda$ des Betongefüges, bestehend aus Zementstein, Gesteinskörnung sowie dem Porenraum mit dem Porenwasser, ist temperaturabhängig. Diese Temperaturabhängigkeit ist unter anderem durch die unterschiedliche Feuchtekonzentration und auch Feuchteverteilung im Betongefüge bei unterschiedlichen Temperaturen begründet. Durch höhere Temperaturen trocknet die Probe, wodurch die Wärmeleitfähigkeit sinkt. Zur Messung dieses Verhaltens wurden zwei Verfahren eingesetzt, das Heizplattenverfahren sowie das Hitzdrahtverfahren [8].
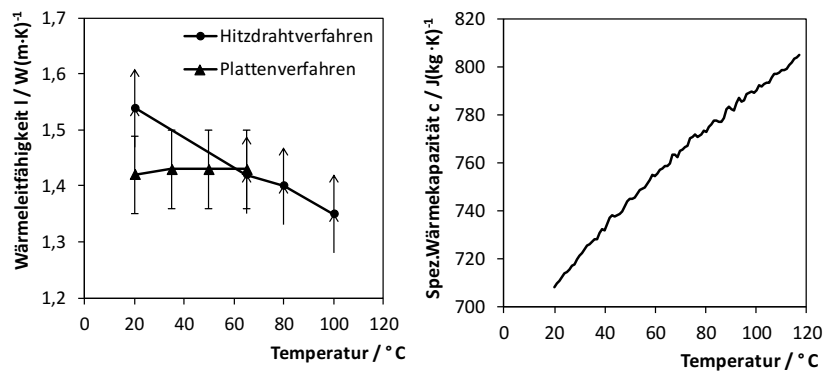

Abb. 1: Links - gemessene spezifische Wärmekapazität; rechts - Wärmeleitfähigkeit des verwendeten Betons.

Fig. 1: Left - measured specific heat capacity; right thermal conductivity of the used concrete.

Die Messung mit dem Heizplattenverfahren ergab eine über die Temperatur weitgehend konstante Wärmeleitfähigkeit von $\lambda=1,43 \pm 0,07 \mathrm{~W}(\mathrm{~m} \cdot \mathrm{K})^{-1}$. Bei der Messung mit dem Hitzdrahtverfahren dagegen wies die Wärmeleitfähigkeit der Betonprobe eine deutliche Temperaturabhängigkeit auf (Abb. 1 links). Bei einer Probentemperatur von $20^{\circ} \mathrm{C}$ wurden $\lambda=1,54 \pm 0,08 \mathrm{~W}(\mathrm{~m} \cdot \mathrm{K})^{-1}$ gemessen, bei $100^{\circ} \mathrm{C}$ nur noch $\lambda=1,35 \pm 0,08 \mathrm{~W}(\mathrm{~m} \cdot \mathrm{K})^{-1}$. Die dazwischen liegenden Messungen deuten auf einen annähernd linearen temperaturabhängigen Verlauf hin. Aufgrund der kurzen Messdauer beim Hitzdrahtverfahren wird der Einfluss der Feuchtigkeit auf die Wärmeleitfähigkeit mitgemessen. Im Gegensatz dazu wird bei der Plattenapparatur durch die längere Messdauer
Feuchtigkeit in der Probe umgelagert, wodurch die gemessene Wärmeleitfähigkeit beeinflusst wird [8]. Daher werden für die Berechnungen in Kapitel 5 die mit dem Hitzdrahtverfahren bestimmten, temperaturabhängigen Wärmeleitfähigkeiten verwendet.

\subsection{Spezifische Wärmekapazität}

Die spezifische Wärmekapazität wurde an zwei Proben über eine temperaturmodulierte dynamische Differenzkalorimetrie (MDSC) ermittelt. Abb. 1 rechts zeigt, dass die spez. Wärmekapazität $\mathrm{c}$ mit der Temperatur ansteigt. Bei einer Probentemperatur von $20^{\circ} \mathrm{C}$ ist $\mathrm{c}=710 \mathrm{~J}(\mathrm{~kg} \cdot \mathrm{K})^{-1}$, steigt bei $80^{\circ} \mathrm{C}$ aber auf ca. $770 \mathrm{~J}(\mathrm{~kg} \cdot \mathrm{K})^{-1}$ [8]. Aufgrund des versuchsrelevanten Temperaturbereichs $\left(20^{\circ} \mathrm{C}-45^{\circ} \mathrm{C}\right.$, siehe Kap. 3) und der relativ geringen Schwankungsbreite wurde ein Wert von $725 \mathrm{~J}(\mathrm{~kg} \cdot \mathrm{K})^{-1}$ (entspricht $33^{\circ} \mathrm{C}$ ) zur Simulation der Erwärmung (Kap. 5) gewählt.

\subsection{Temperaturausdehnungskoeffizient}

Der Temperaturausdehnungskoeffizient von Beton $\alpha_{T, B}$ wird maßgeblich durch das Ausdehnungsverhalten der Gesteinskörnung, der Matrix, dem anteiligen Volumen von Gesteinskörnung und Matrix sowie dem Feuchtigkeitsgehalt bestimmt. Im Rahmen der Voruntersuchungen wurde mit einem Dilatometer in einem Temperaturbereich von $20^{\circ} \mathrm{C}$ bis $80^{\circ} \mathrm{C}, \alpha \mathrm{T}, \mathrm{B}$ mit $(0,010 \pm 0,001) \mathrm{mm}(\mathrm{K} \cdot \mathrm{m})^{-1}$ bestimmt. Eine signifikante Temperaturabhängigkeit wurde nicht festgestellt. Mit dem in der Literatur für Normalbeton angegebenen Wert $\alpha_{T, B}=0,01 \mathrm{~mm}(\mathrm{~K} \cdot \mathrm{m})^{-1}$ [9] stimmt der gemessene gut überein. In einem weiteren Schritt wird bei späteren Berechnungen der Temperaturausdehnungskoeffizient verwendet, um die inneren Spannungen in dem Betonprobekörper aufgrund von Temperaturunterschieden (simulierte Temperaturfelder) zu bestimmen. Diese Ergebnisse geben dann weiter Aufschluss zu den Versagensmechanismen in der Probe.

\section{Messungen während dynamischer Belastung}

Am Institut für Massivbau an der Leibniz Universität Hannover wurden an 27 zylindrischen Betonprobekörper dynamische Druckschwellversuche durchgeführt. In der hier beschriebenen Versuchsreihe wurden Zylinder mit einem Durchmesser von $\mathrm{d}=100 \mathrm{~mm}$ und einer Höhe von $\mathrm{h}=300 \mathrm{~mm}$ untersucht. Die Zusammensetzung des Probekörperbetons ist in [7] 
beschrieben. Für die dynamische Beanspruchung der Proben kam eine servohydraulische Universalprüfmaschine zum Einsatz. Während der dynamischen Druckversuche wurden an einer Vielzahl von Messstellen die Luft-, Oberflächen- und Kerntemperaturen gemessen. Weiterhin wurden die von den Druckplatten zurückgelegten Wegstrecken bei der Belastung des Zylinders mit Laserdistanzsensoren aufgezeichnet und daraus die Dehnungen im Beton berechnet. Simultan wurde die aufgebrachte Spannung aus der eingestellten Belastung und der Zylinderquerschnittsfläche aufgezeichnet und berechnet. In [7] konnte durch die Analyse von Spannungs-Dehnungs-Diagrammen gezeigt werden, dass während der Versuche Energie vom Probekörper aufgenommen wird und in diesem verbleibt. Der Vergleich dieser experimentell ermittelten Dissipationsenergie und der Erwärmung der Betonprobe zeigte eine deutliche Korrelation.

\subsection{Messaufbau}

Zur Messung der Betontemperaturen sowie Umgebungstemperaturen wurden 19 Thermoelemente Typ T (absolute Genauigkeit $\Delta \mathrm{T}=0,1 \mathrm{~K}$ ) verwendet ( $\mathrm{Abb}$. 2). Die Thermosensoren im Probekörper wurden in vorgebohrte Löcher auf der Symmetrieachse des Zylinders eingeleimt $(5 \mathrm{~cm}$ senkrechter Abstand zur Manteloberfläche), sodass die Kerntemperaturen in verschiedenen Höhen (mittig sowie $5 \mathrm{~cm}$ über und unter der Mitte) gemessen werden konnten. Weitere Temperatursensoren befanden sich auf der Zylinderoberfläche, an den Druckplatten, auf der Plexiglasscheibe (Splitterschutz) sowie in der Luft im Abstand von $1 \mathrm{~mm}$ und $50 \mathrm{~mm}$ von der Zylinderoberfläche. Zur Untersuchung der Wärmeentwicklung der Betonproben wurden neben Thermoelementen zur Oberflächentemperaturmessung auch Thermografie eingesetzt.

\subsection{Messregime/Randbedingungen}

Die Betonproben wurden in der Prüfmaschine mit einer sinusförmigen Belastung (Wiederholfrequenz der Beanspruchung / Belastungsfrequenz $2 \mathrm{~Hz}$ und $8 \mathrm{~Hz})$ bis zum Bruch beansprucht. Das Oberspannungsniveau betrug $60 \%$ bis $80 \%$ der statischen Bruchlast, das Unterspannungsniveau $5 \%$. Es wurden je Lastregime 3 Probekörper geprüft. Die untere Druckplatte der Prüfmaschine wurde durch eine Plattenkühlung auf $22^{\circ} \mathrm{C}$ gekühlt, um einer prüfmaschinenbedingten Erwärmung der Probekörper entgegen zu wirken. Die Lufttemperatur in der weiteren Umgebung der Prüfmaschine war während der Prüfung weitestgehend konstant $\left(18^{\circ} \mathrm{C}-20^{\circ} \mathrm{C}\right)$.

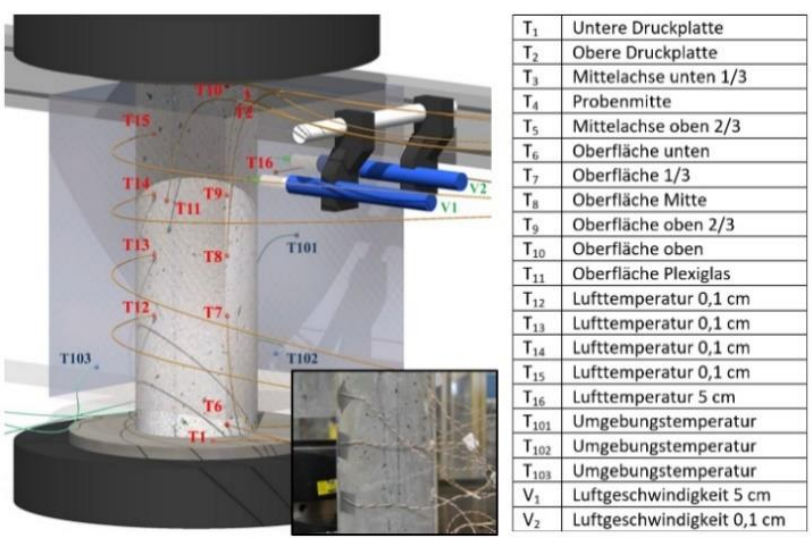

Abb. 2: Messaufbau zur Messung von Kern-, Oberflächen- und Lufttemperaturen sowie Strömungsgeschwindigkeit.

Fig. 2: Measurement setup for measuring core, surface, and air temperatures as well as flow speed.

\section{Messergebnisse}

Im Folgenden sind die Messergebnisse exemplarisch für eine typische Probe mit stetig steigender Temperatur und Bruch am Ende des Versuchs anhand der Diagramme in Abb. 3 beschrieben. Die Belastungsfrequenz dieser Probe betrug $8 \mathrm{~Hz}$ und das Oberspannungsniveau $S_{0} 65 \%$ der Bruchlast. Der Bruch der Probe erfolgte nach 136.144 Lastwechseln bzw. 4,75 h.

Das Temperaturmaximum von $47^{\circ} \mathrm{C}$ liegt - wie auch bei anderen Proben - in der oberen Hälfte des Zylinders (Abb. 3a). Mögliche Ursachen für die asymmetrische Temperaturverteilung in der Probe sind der Wärmetransport vom Betonzylinder $\left(\mathrm{T}_{6}, \mathrm{~T}_{10}\right)$ in die unterschiedlich temperierten Druckplatten sowie eine ungleichmäßige Energiedissipation im Probekörper. Das gleiche Verhalten ist bei den Oberflächentemperaturen zu beobachten (Abb. 3b). Abb. 3c zeigt die Lufttemperaturen direkt neben der Betonoberfläche und Abb. $3 \mathrm{~d}$ die Temperaturen am Splitterschutz und in der Umgebungsluft im Vergleich zu Kern- und Oberflächentemperatur. Es zeigt sich dabei, dass die Luft um den Zylinder innerhalb des Splitterschutzes sich erwärmt, was bei den Simulationen in Kapitel 5 berücksichtigt wird. 


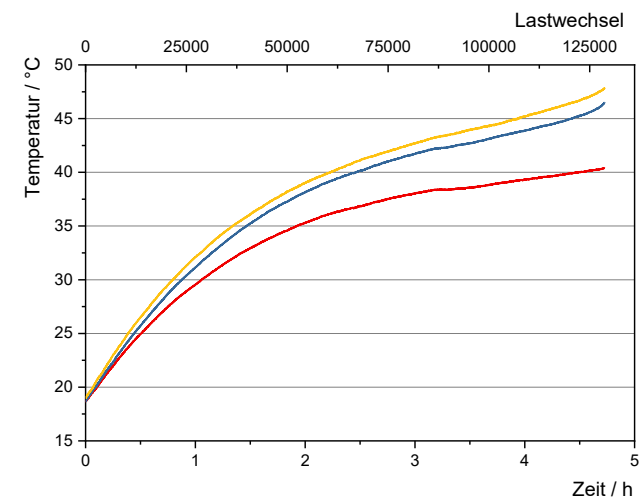

a) Kerntemperaturen

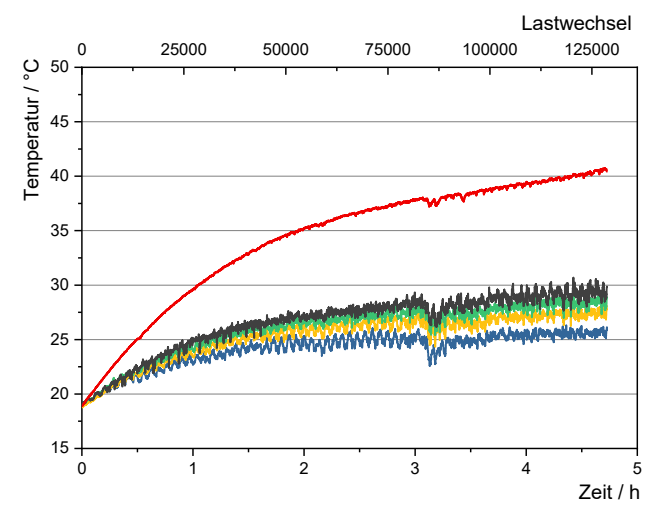

c) Lufttemperaturen in Abstand d $=1 \mathrm{~mm}$ von Probenoberfläche ( $T_{8}$ - Oberflächentemperatur Mitte)
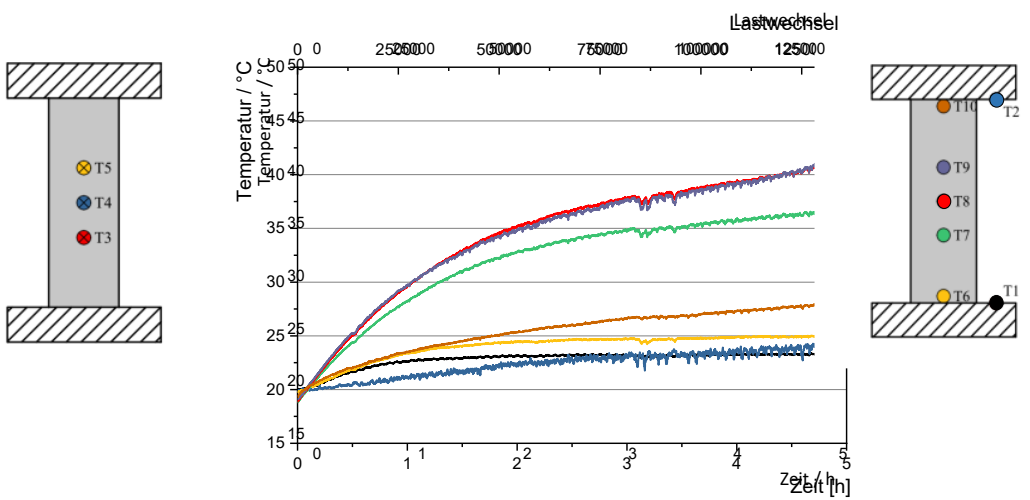

b) Oberflächentemperaturen

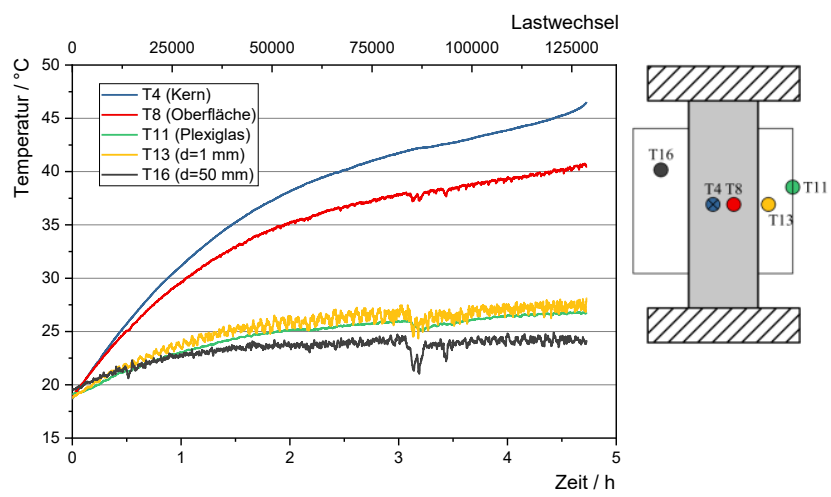

d) Vergleich der Umgebungstemperaturen: Splitterschutz $(d=2 \mathrm{~mm})$, Luft ( $d=10 \mathrm{~mm}$ und $d=50 \mathrm{~mm}) ;$ zusätzlich Kern und Oberfläche

Abb. 3 (a-d): Probe ZA 2.10*; $f_{p}=8 \mathrm{~Hz} ; S_{o}=65 \%$; zeitlicher Temperaturverlauf aller Temperaturmesspunkte (Betonkern $\left(T_{3}-T_{5}\right)$, Betonoberfläche $\left(T_{6}-T_{10}\right)$, Druckplatten $\left(T_{1}, T_{2}\right)$, Luft $\left(T_{12}-T_{16}\right)$, Splitterschutz $\left(T_{11}\right)$.

*(Probekörperbezeichnung ZA X.X wurde von der Versuchsdurchführung übernommen)

Fig. $3(a-d)$ : Sample ZA $2.10 * ; f_{p}=8 \mathrm{~Hz} ; S_{o}=65 \%$; temporal temperature profile of all temperature measuring points (concrete core $\left(T_{3}-T_{5}\right)$, concrete surface $\left(T_{6}-T_{10}\right)$, pressure plates $\left(T_{1}, T_{2}\right)$, air $\left(T_{12}-T_{16}\right)$, splinter protection $\left(T_{11}\right)$.

* (Specimen designation ZA X.X was adopted from the test procedure)
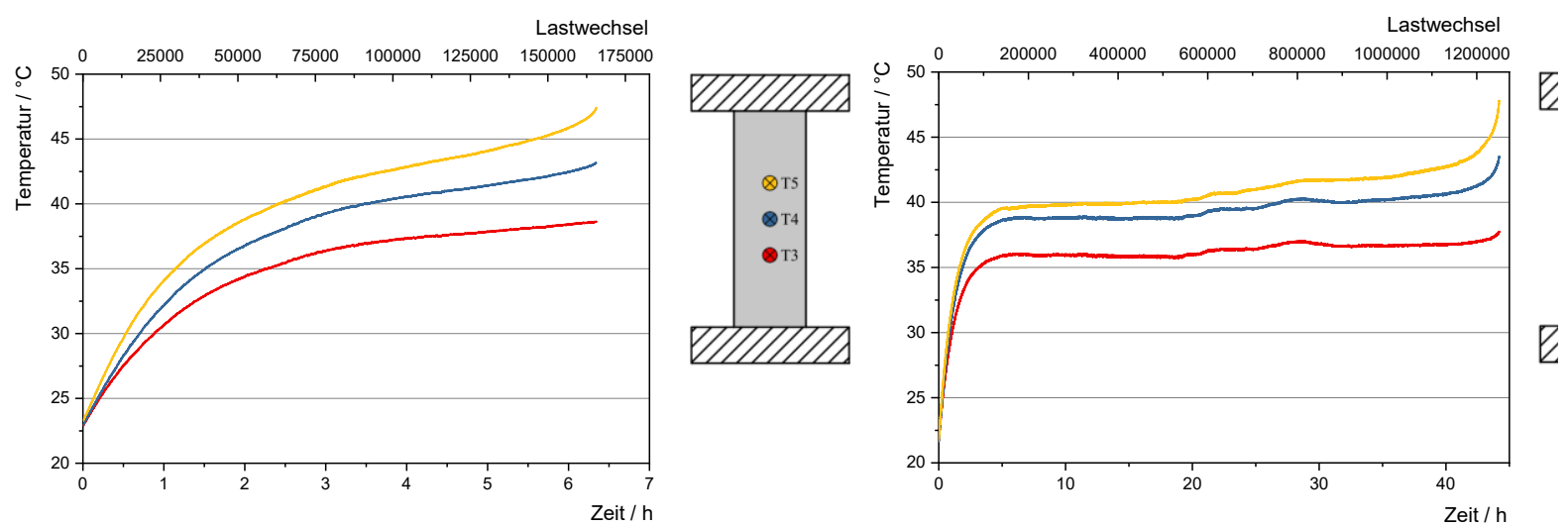

Abb. 4: $f_{p}=8 \mathrm{~Hz} ; S_{o}=60 \%$; zeitlicher Temperaturverlauf im Zylinderkern;

links - Probe ZA 2.11; rechts - ZA 3.1.

Fig. 4: $f_{p}=8 \mathrm{~Hz} ; S_{o}=60 \%$; temporal temperature profile in the cylinder core;

left - sample ZA 2.11; right - ZA 3.1 
Vergleicht man die Temperaturverläufe identischer Versuche (gleiche Belastungsfrequenz und Oberspannung), zeigen sich mitunter deutliche Unterschiede bei dem Versagenszeitpunkt und bei dem Temperaturverlauf (Abb. 4). Diese Unterschiede können auf Inhomogenitäten in der Zylinderprobe zurückgeführt werden und konnte ebenfalls bei anderen Probekörpern und Belastungen beobachtet werden.

Der Temperaturunterschied über die Probenhöhe in Abb. 4 stellt sich schon kurz nach Versuchsbeginn ein und nimmt bis zum Ende hin zu. Kurz vor dem Versagen des Probekörpers, wenn die Schädigungen im Betongefüge zum Bruch führen, steigt im wärmeren Bereich die Temperatur noch einmal deutlich an (Messpunkt $\mathrm{T}_{5}$ ). Dieser Temperaturanstieg ist dabei größer als im kühleren Bereich $\left(\mathrm{T}_{3}\right)$, was auf eine größere, freigesetzte Dissipationsenergie schließen lässt. Interessanterweise werden bei beiden Versuchen, trotz völlig unterschiedlicher Lastwechselzahlen/Laufzeiten, ähnliche Temperaturen erreicht.

Das Temperaturprofil am Ende eines Druckversuches zeigt prinzipiell deutliche Temperaturunterschiede, wobei das Temperaturmaximum (Hotspot) meist oberhalb der Probenmitte (Abb. 5 links), vereinzelt aber auch darunter liegen kann (Abb. 5 rechts). Dieses Verhalten ist sowohl bei kürzeren als auch bei längeren Versuchsdauern zu beobachten. Da bei allen Zylindern genau am Temperaturhotspot das (spätere) Schadenszentrum liegt, steht die Temperaturverteilung in der Probe im Zusammenhang mit dem Schädigungsprozess [7].

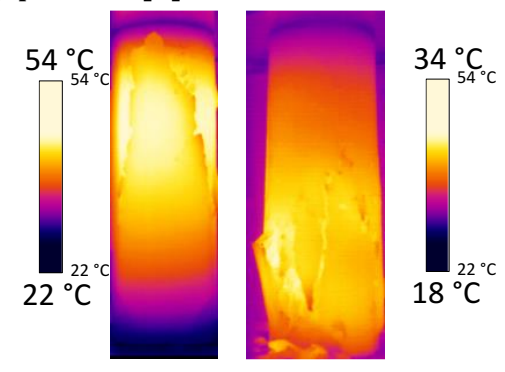

Abb. 5: Thermogramm Probe ZA 2.11 (links) und ZA 3.9 (rechts) nach Versagenseintritt.

Fig. 5: Thermogram of the sample ZA 2.11 (left) and ZA 3.9 (right) after failure has occurred.

\subsection{Zusammenfassung der Messungen}

Abb. 6 zeigt für alle durchgeführten Messreihen (Belastungsfrequenzen von 2 und $8 \mathrm{~Hz}$ ) den Mittelwert der Temperaturerhöhung sowie die dazugehörigen

Standardabweichungen als Maß für die Streuung. Es wird deutlich, das mit steigendem Beanspruchungsniveau die Probekörpererwärmung sinkt. Grund dafür ist das zeitige Versagen des Probekörpers bei hoher Belastung und der damit einhergehenden kürzeren Erwärmungszeit. Außerdem zeigt der Vergleich beider Diagramme, dass eine höhere Belastungsfrequenz erwartungsgemäß zu höheren Temperaturen führt, da mehr Energie im selben Zeitraum dissipiert wird. Die relativ großen Streubreiten der maximalen Erwärmung können auf die Inhomogenität des Betons und unterschiedliche Anfangsrissbildung im Gefüge zurückgeführt werden.

Belastungsfrequenz $f_{p} \quad \mathbf{2} \mathbf{H z}$
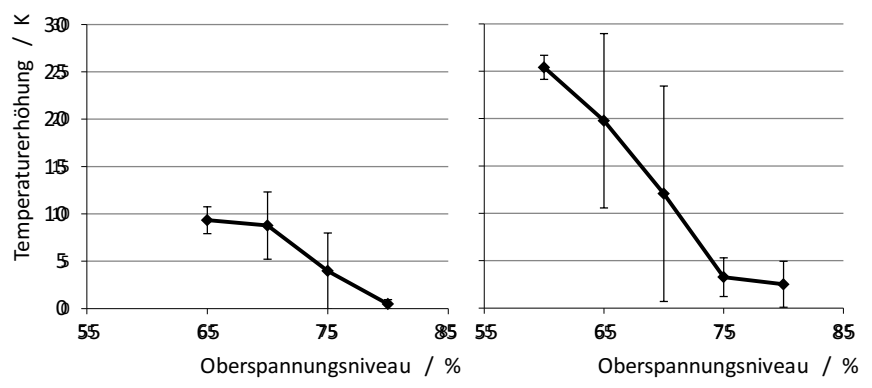

Abb. 6: Temperaturerhöhung im Kern der Betonproben Mittelwert für gleiche Prüfbedingungen in Abhängigkeit von der Belastungsstärke $S_{o}$; Standardabweichung als Maß für die Streubreite.

Fig. 6: Temperature increase in the core of the concrete samples - average value for the same test conditions depending on the load intensity $S_{o}$; Standard deviation as a measure of the spread.

\subsection{Mathematische Beschreibung der Wärmeentwicklung}

Aus den in [7] ausgewerteten Hysteresekurven der Dehnungen wurde für jeden Versuch die zeitabhängige Dissipationsenergie berechnet. Für die große Anzahl an zum Teil stark schwankenden Messwerten sind durch nichtlineare Approximation Funktionen gefunden worden, die den zeitlichen Verlauf der im Probekörper freigesetzten Energie beschreiben. Als beschreibende Funktionen dienen hier Polynome 6. bis 9. Grades, die eine gute Übereinstimmung mit den Messwerten zeigen (Mittelwert aller Korrelationsfaktoren 0,93). Die approximierten Kurven für die Beschreibung der frei werdenden Dissipationsenergie gehen dann in die Simulation der Probekörpererwärmung als Heizrate ein. 


\section{Simulation der Temperaturentwicklung}

Um Rückschlüsse auf die Ursachen und Verläufe der Betonerwärmung ziehen zu können, wurde die Erwärmung der Proben mit dem Finite-ElementeProgramm ANSYS berechnet.

\subsection{Modellierung und Randbedingungen}

Die Modellierung der zylinderförmigen Betonprobe sowie die thermischen Umgebungsbedingungen und Materialkoeffizienten sowie Simulationsbedingungen sind in

Tab. 1 aufgeführt. Für den Grenzbereich BetonLuft wurde ein mittlerer Wärmeübergangskoeffizient von $\alpha_{\text {Luft }}=5,5 \mathrm{~W} /\left(\mathrm{m}^{2} \cdot \mathrm{K}\right)$ errechnet. Dafür wurden neben einer analytischen Ermittlung über eine Mischkonvektion an der Grenzschicht Oberfläche Zylinder - Luft auch eine experimentelle Bestimmung über Anemometer, Wärmestrommessplatten und Temperatursensoren an einem künstlich erwärmten Zylinder durchgeführt. Da die Druckplatten der Prüfmaschine an die Ober- und Unterseite des Zylinders grenzen, erfolgt hier der Wärmeübergang nur durch Leitung und ist somit bedeutend größer als von der Zylinderoberfläche an die Umgebung (Konvektion und Strahlung), was durch einen Wärmeübergangskoeffizienten $\quad \alpha_{\text {Stahl }}=155 \mathrm{~W} /\left(\mathrm{m}^{2} \cdot \mathrm{K}\right)$ berücksichtigt wurde [10]. Als Quelle für die Erwärmung diente bei den Simulationen die aus den Spannungs-Dehnungslinien ermittelte
Dissipationsenergie [7]. Auf der Modellannahme beruhend, dass jedes Element die gleiche Dehnung erfährt, wird im Simulationsmodell jedem Element zeitlich abhängig die gleiche Energie zugeführt. Einziger Freiheitsgrad während der Simulation ist somit nur die Betontemperatur.

Tab. 1: Simulationsparameter.

Tab. 1: Simulations parameters.

\begin{tabular}{|c|c|}
\hline $\begin{array}{l}\quad \text { Geometrie } \\
\text { Zylinder } \\
-\mathrm{h}=300 \mathrm{~mm} \\
-\mathrm{d}=100 \mathrm{~mm} \\
-\quad \text { als,,solid body“ } \\
\text { definiert } \\
\text { (Volumenkörper) }\end{array}$ & $\begin{array}{l}\quad \text { Material } \\
\text { Beton } \\
\text { - Rohdichte } \rho=2125 \mathrm{~kg} / \mathrm{m}^{3} \text { (Messwert) } \\
\text { - Wärmeleitfähigkeit } \\
\quad \lambda(\mathrm{T})=(1,48-1,54) \mathrm{W} /(\mathrm{m} \cdot \mathrm{K}) \\
\text { - } \begin{array}{l}\text { Spez. Wärmekapazität } \mathrm{c}=725 \\
\mathrm{~J} /(\mathrm{kg} \cdot \mathrm{K})\end{array}\end{array}$ \\
\hline $\begin{array}{l}\text { Vernetzung } \\
\text { - } \text { Netztyp Hexaeder } \\
\text { - } 5209 \text { Knoten } \\
\text { - } 1080 \text { Elemente } \\
\text { - Regelmäßige } \\
\text { Element- } \\
\text { Verteilung } \\
\end{array}$ & $\begin{array}{l}\quad \text { Analyse } \\
\text { - Transient; } 100 \text { Lastschritte } \\
\text { á } 1 / 100 \text { x Gesamtdauer } \\
\text { - Solvertyp: iterativ } \\
\text { - Konvergenztoleranz: } 0,001 \\
\text { - Max. Anzahl Iterationen: } 100 \\
\text { - } \text { Solver-Toleranz: } 0,1 \mathrm{~W} / \mathrm{m}^{2} \\
\end{array}$ \\
\hline $\begin{array}{r}\text { Umgebungst } \\
\text { - Anfangstemperatur } \\
\text { - Wärmeübergang de } \\
\text { - Oben und unten zu } \\
\text { - Mantelfläche zu Lu } \\
\text { - Umgebungstempera } \\
\text { zeitabhängige Funk } \\
\text { - Interne Heizrate als } \\
\text { dissipierten Energie }\end{array}$ & $\begin{array}{l}\text { edingungen } \\
\text { robekörperspezifisch }(19,1-23,0){ }^{\circ} \mathrm{C} \\
\text { Außenflächen: } \\
\text { tahl } \alpha_{\text {Stahl }}=155 \mathrm{~W} /\left(\mathrm{m}^{2} \cdot \mathrm{K}\right) \\
\alpha_{\text {Luft }}=5,5 \mathrm{~W} /\left(\mathrm{m}^{2} \cdot \mathrm{K}\right) \\
\text { uren }(\text { Luft, Stahlplatten) sind } \\
\text { onen, basierend auf Messwerten } \\
\text { eitabhängige Funktion basierend auf der } \\
\text { oei der Dehnungshysterese }\end{array}$ \\
\hline
\end{tabular}



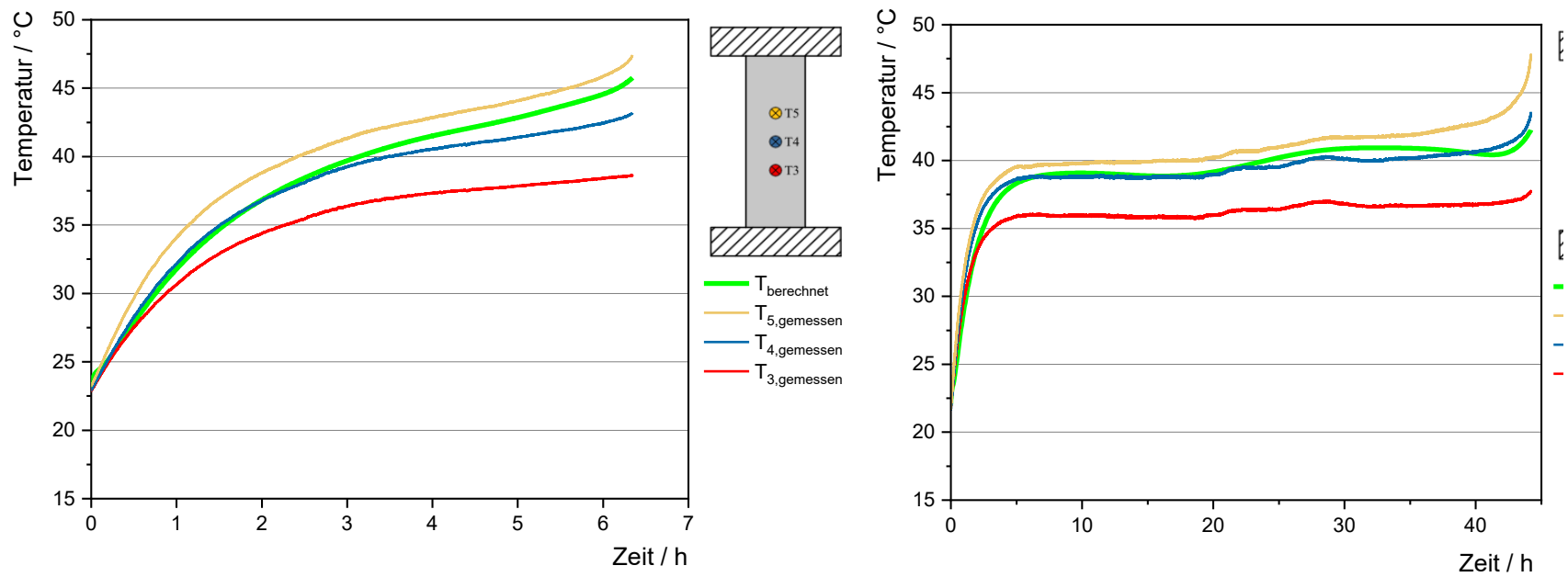

Abb. 7: $f_{p}=8 \mathrm{~Hz} ; S_{o}=60 \%$; zeitlicher Temperaturverlauf im Zylinderkern berechnet (1,7 cm oberhalb des Kerns) und gemessen; links - Probe ZA 2.11; rechts - Probe ZA 3.1.

Fig. 7: $f_{p}=8 \mathrm{~Hz} ; S_{o}=60 \%$; temporal temperature profile in the cylinder core calculated $(1.7 \mathrm{~cm}$ above the core) and measured; left - sample ZA 2.11; right-sample ZA 3.1.

Tab. 2: Vergleich der gemessenen und simulierten maximalen Betonkerntemperaturen bei Versuchsende (Versagen oder Abbruch; Versuchsdauer $>20 \mathrm{~min}$ ).

Tab. 2: Comparison of the measured and simulated maximum concrete core temperatures at the end of the test (failure or termination; test duration $>20 \mathrm{~min}$ ).

\begin{tabular}{|c|c|c|c|c|c|c|c|}
\hline $\begin{array}{c}\text { Probe- } \\
\text { körper }\end{array}$ & $\begin{array}{c}\text { Simulationsdauer } \\
{[\mathbf{d}: \mathbf{h : m}]}\end{array}$ & $\begin{array}{c}\text { Last- } \\
\text { wechsel } \\
\text { N [-] }\end{array}$ & $\begin{array}{c}\text { Spannungs- } \\
\text { niveau } \\
\mathrm{S}_{\text {in }}[\mathbf{\%}] \mathrm{d} . \\
\text { Bruchlast }\end{array}$ & $\begin{array}{c}\text { Belastungs- } \\
\text { frequenz } \mathrm{f}_{\mathrm{p}} \\
{[\mathbf{H z}]}\end{array}$ & $\begin{array}{c}\mathrm{T}_{\text {simuliert }} \\
{\left[{ }^{\circ} \mathbf{C}\right]}\end{array}$ & $\begin{array}{c}\mathrm{T}_{\text {gemessen }} \\
{\left[{ }^{\circ} \mathbf{C}\right]}\end{array}$ & $\begin{array}{c}\Delta \mathrm{T}= \\
\mathrm{T}_{\text {sim- }} \\
\mathrm{T}_{\text {mess }} \\
{[\mathbf{K}]}\end{array}$ \\
\hline ZA 4.4 & $0: 13: 53$ & $100 \mathrm{k}$ & 75 & 2 & 32,0 & 30,1 & 1,9 \\
\hline ZA 1.7 & $0: 00: 40$ & $19 \mathrm{k}$ & 70 & 8 & 30,8 & 31,8 & $-1,0$ \\
\hline ZA 4.5 & $0: 07: 10$ & $210 \mathrm{k}$ & 70 & 8 & 43,7 & 44,2 & $-0,5$ \\
\hline ZA 1.8 & $0: 09: 19$ & $67 \mathrm{k}$ & 70 & 2 & 34,2 & 32,8 & 1,4 \\
\hline ZA 2.8 & $0: 02: 29$ & $15 \mathrm{k}$ & 70 & 2 & 30,4 & 27,9 & 2,5 \\
\hline ZA 2.9 & $0: 01: 00$ & $7,2 \mathrm{k}$ & 70 & 2 & 25,4 & 25,2 & 0,2 \\
\hline ZA 2.10 & $0: 04: 44$ & $140 \mathrm{k}$ & 65 & 8 & 46,0 & 47,9 & $-1,9$ \\
\hline ZA 3.9 & $0: 00: 26$ & $13 \mathrm{k}$ & 65 & 8 & 27,4 & 29,9 & $-2,5$ \\
\hline ZA 1.10 & $1: 10: 43$ & $1.000 \mathrm{k}$ & 65 & 8 & 38,8 & 40,1 & $-1,3$ \\
\hline ZA 3.8 & $5: 18: 53$ & $1.000 \mathrm{k}$ & 65 & 2 & 27,9 & 28,9 & $-1,0$ \\
\hline ZA 4.6 & $5: 18: 53$ & $1.000 \mathrm{k}$ & 65 & 2 & 28,8 & 28,6 & 0,2 \\
\hline ZA 1.2 & $2: 05: 36$ & $390 \mathrm{k}$ & 65 & 2 & 31,2 & 30,4 & 0,8 \\
\hline ZA 3.1 & $1: 20: 12$ & $1.300 \mathrm{k}$ & 60 & 8 & 40,5 & 43 & $-2,5$ \\
\hline ZA 2.11 & $0: 06: 21$ & $180 \mathrm{k}$ & 60 & 8 & 45,8 & 47,4 & $-1,6$ \\
\hline
\end{tabular}

Tab. 2 zeigt zusammenfassend die gemessenen sowie die berechneten maximalen Kerntemperaturen in den Betonproben. Der Vergleich zeigt, dass der überwiegende Teil der Ergebnisse sehr gut übereinstimmt. Die Abweichungen betragen bis zu
$\Delta \mathrm{T}= \pm 2,5 \mathrm{~K}$, der Mittelwert aller Abweichungen (Beträge) zwischen Simulation und Messung ergibt sich zu 1,4 K. Vergleicht man die gemessenen und berechneten maximalen Oberflächentemperaturen auf dem Zylinder, ergibt sich eine mittlere Abweichung von 
1,5 K. Da insgesamt alle Temperaturunterschiede zwischen Messung und Simulation relativ klein sind, können die Simulationen der Temperaturentwicklung der sich erwärmenden Zylinder durch die Messungen als validiert angesehen werden.

Die Abweichungen zwischen Simulation und Messung können vor allem auf das inhomogene Materialverhalten des Betons zurückgeführt werden. So kann beispielsweise das Temperaturmaximum (Hotspot) während der Messung, je nach Vorschädigung und Ort der inertialen Rissbildung, an verschiedenen Stellen entstehen, wohingegen die berechneten Temperaturmaxima stets oberhalb der Zylindermitte liegen. Da die Lage des Hotspots nicht vorhergesagt werden kann, die Temperatursensoren aber eine feste Lage im Zylinder haben, kann weiterhin die gemessene Kerntemperatur an den Messpunkten etwas von der tatsächlichen Maximaltemperatur abweichen.

\section{Zusammenfassung und Ausblick}

An 27 Prüfkörpern aus Beton wurden dynamische Druckschwellenversuche durchgeführt und die dabei gemessenen Probekörpertemperaturen mit Simulationen verglichen. Für die Simulation der Probekörpertemperatur wurde die experimentell ermittelte Dissipationsenergie [7] verwendet. Die so berechneten maximalen Kerntemperaturen stimmen gut mit den gemessenen Werten überein. Die Hypothese, dass es sich bei der Dissipationsenergie um die für die Probekörpererwärmung verantwortliche thermische Energie handelt, konnte bestätigt werden. In einem nächsten Schritt sollen die bereits gemessenen Lufttemperaturen sowie -geschwindigkeiten mit CFDSimulationen unter Berücksichtigung analytisch berechneter Wärmeübergangswiderstände verglichen werden. Ziel ist es, nicht nur die Wärmeentstehung besser zu verstehen, sondern auch geeignete Maßnahmen zur Temperaturbegrenzung abzuleiten.

\section{Danksagung}

Die Autoren bedanken sich bei der Deutschen Forschungsgemeinschaft für die Förderung des Projekts „Ursachen und Modellierung der Erwärmung von ermüdungsbeanspruchten Betonproben“ DFGProjektnummer 342801069. Dank gilt auch Dominik Bock, dessen ausgezeichnete Bachelorarbeit die Auswertung der Temperaturmessungen zum Thema hatte.

\section{Literatur}

[1] Elsmeier, K.: Influence of temperature on the fatigue behaviour of concrete. In: fib Symposium Copenhagen, Denmark (2015).

[2] von der Haar C, Hümme J, Marx S, Lohaus L: Untersuchungen zum Ermüdungsverhalten eines höherfesten Normalbetons. In: Beton- und Stahlbetonbau 110(10) (2015) p.699-709.

[3] Phan, L. T.; Carino, N. J.: Code provisions for high strength concrete strength-temperature relationship at elevated temperatures. In: Materials and Structures 36, (2003) p. 91-98.

[4] Voelker, C.; Diewald, M.: CFD simulation and measurement of the heat transfer from building material specimens to the indoor environment. In: Building Simulation International Conference of the International Building Performance Simulation Association. Hyderabad, India, (2015) p. 891-896.

[5] Diewald, M.; Voelker, C.: Heat transfer from building material probes to the indoor environment. In: Roomvent International Conference on Air Distribution in Rooms. Sao Paulo, Brasil, (2014) p. 633-640.

[6] Bode, M.; Marx, S.: Heat Generation during fatigue tests on concrete specimens. In: fib Symposium Kraków, Poland (2019).

[7] Bode, M.; Marx, S.; Vogel, A.; Völker, C.: Dissipationsenergie bei Ermüdungsversuchen an Betonprobekörpern. In: Beton- und Stahlbetonbau 114 (2019) p. 548-556.

[8] Hemberger, F.: Thermische Charakterisierung einer Betonprobe. Report ZAE 2-0518-01. Bayerisches Zentrum für Angewandte Energieforschung e.V (2018).

[9] Deutsches Institut für Normung: Eurocode 2: Bemessung und Konstruktion von Stahlbeton- und Spannbetontragwerken - Teil 1-1: Allgemeine Bemessungsregeln und Regeln für den Hochbau (DIN EN 1992-1-1). Beuth Verlag (2011).

[10] von der Haar C.; Wedel F.; Marx S.: Numerical and experimental investigations of the warming of fatigue-loaded concrete. In: fib Symposium Cape Town, South Africa (2016). 